\title{
Role of High-Resolution Magnetic Resonance Imaging in the Diagnosis of Primary Angiitis of the Central Nervous System
}

\author{
Hyun Jin Noh, ${ }^{a}$ Jin Wook Choi, ${ }^{b}$ Jun Pyo Kim, ${ }^{a}$ Gyeong Joon Moon, ${ }^{c}$ Oh Young Bang ${ }^{a}$ \\ ${ }^{\mathrm{a}}$ Departments of Neurology and ${ }^{\mathrm{b}}$ Radiology, Samsung Medical Center, Sungkyunkwan University School of Medicine, Seoul, Korea \\ ${ }^{\circ}$ Clinical Research Center, Samsung Biomedical Research Institute, Seoul, Korea
}

Received December 1, 2012

Revised May 2, 2013

Accepted May 3, 2013

\section{Correspondence}

Oh Young Bang, MD, PhD

Department of Neurology,

Samsung Medical Center,

Sungkyunkwan University

School of Medicine,

81 Irwon-ro, Gangnam-gu,

Seoul 135-710, Korea

Tel $+82-2-3410-3599$

Fax $+82-2-3410-0052$

E-mailnnmboy@unitel.co.kr
Background Primary angiitis of the central nervous system (PACNS) is a rare disorder and is often difficult to diagnose due to the lack of a confirmatory test. PACNS can generally be diagnosed based on typical angiographic findings. We describe herein a patient diagnosed with PACNS despite the presence of normal findings on conventional angiography.

Case Report A 44-year-old man with a recent history of ischemic stroke in the right posterior cerebral artery territory developed acute-onset vertigo. Diffusion-weighted imaging revealed an acute infarction within the left posterior inferior cerebellar artery. His medical history was unremarkable except for hyperlipidemia; the initial examination revealed mild gait imbalance. During the 10 days of hospital admission, the patient experienced four recurrent ischemic strokes within the posterior circulation territory (occipital lobe, pons, and cerebellum). He was diagnosed with recurrent cerebral infarctions due to PACNS. The basilar artery exhibited no demonstrable luminal stenosis, but there were direct imaging signs of central nervous system angiitis including wall thickening and contrast enhancement. High-dose intravenous steroid therapy followed by oral prednisolone was administered. There was no further stroke recurrence and follow-up imaging of the arterial walls showed normalization of their characteristics.

Conclusions The present case emphasizes the importance of wall imaging in the diagnosis and treatment of PACNS.

J Clin Neurol 2014;10(3):267-271

Key Words ischemic stroke, magnetic resonance imaging, vasculitis, inflammation.

\section{Introduction}

Primary angiitis of the central nervous system (PACNS) is characterized by the inflammation and destruction of central nervous system (CNS) vessels without vasculitis outside of the CNS. PACNS is a rare disorder that is poorly understood. The clinical signs are nonspecific and diagnosis is often difficult due to the lack of a confirmatory test. ${ }^{2}$ In general, PACNS can be diagnosed based on typical angiographic findings and by excluding other possible etiologies such as systemic vasculitis, CNS infection, and malignancy. The typical angiographic findings of PACNS include alternating areas of ste-

(a) This is an Open Access article distributed under the terms of the Creative Commons Attribution Non-Commercial License (http://creativecommons.org/licenses/by-nc/3.0) which permits unrestricted non-commercial use, distribution, and reproduction in any medium, provided the original work is properly cited. nosis and dilation, referred to as beading, which can be smooth or irregular and typically occurs bilaterally, but can also include single vessels. We describe herein a patient with recurrent ischemic stroke diagnosed as PACNS who exhibited no abnormal findings on conventional angiography.

\section{Case Report}

A 44-year-old right-handed man experienced a sudden onset of vertigo and nausea while washing his face. The patient had experienced a stroke 3 months previously that had been diagnosed as a left posterior cerebral artery (PCA) infarction (Fig. 1A). The only risk factor he possessed was hyperlipidemia, and his current medications included statin and aspirin. The patient visited the stroke center of a local hospital where a brain magnetic resonance (MR) imaging (MRI) scan revealed diffusion restriction in the left posterior inferior cerebellar 

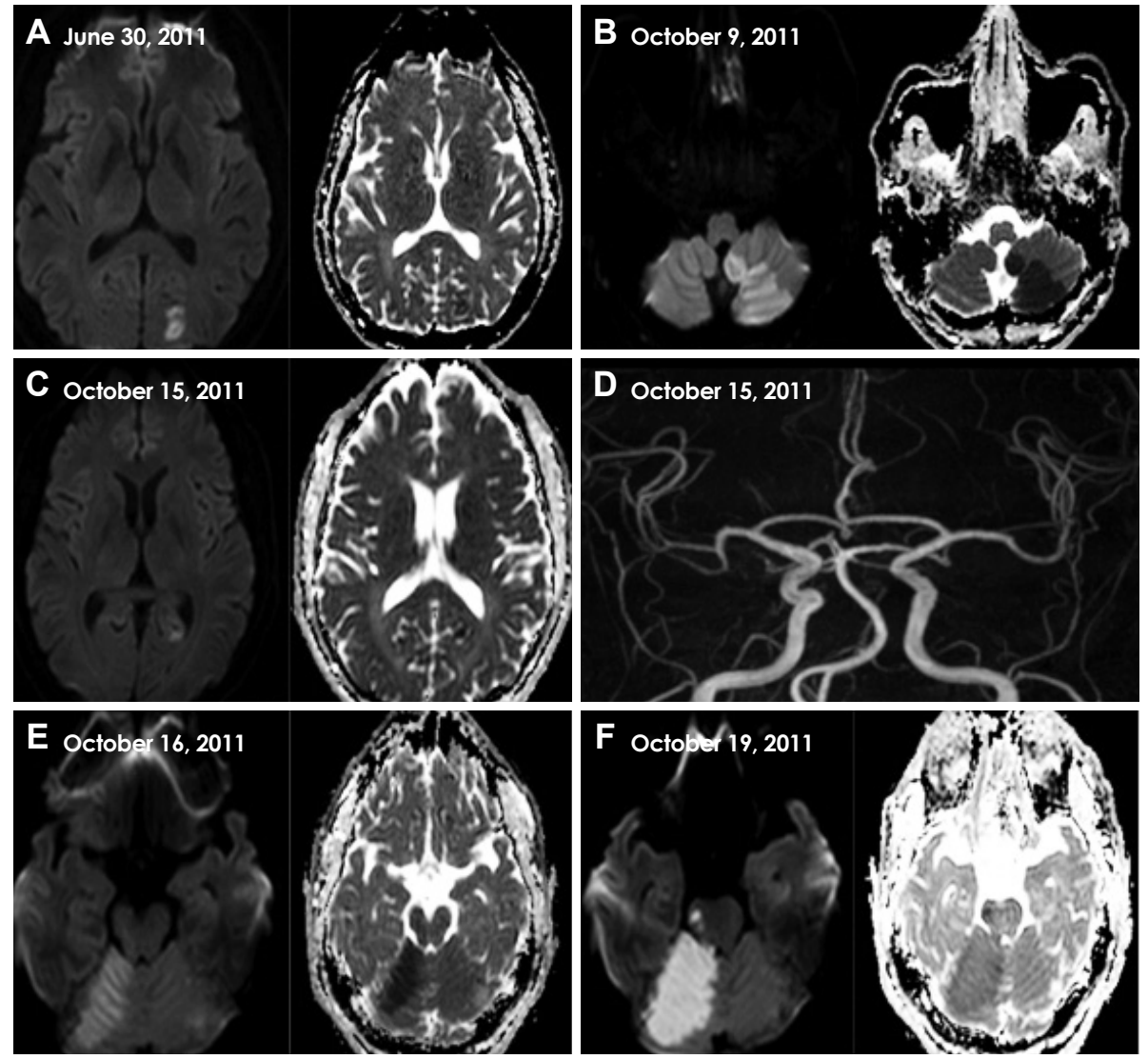

Fig. 1. Serial MRI findings of five recurrent strokes in the present case. A: Three months previous to the current admission, DWI and ADC revealed acute left PCA infarction. B: On day 0, MRI revealed acute left PICA infarction. C: On day 6, the patient had a new ischemic infarction in the left PCA territory. D: MRA revealed normal intracranial arteries including in the vertebrobasilar system. E: On day $7, \mathrm{MRI}$ revealed a new ischemic infarction in the right superior cerebellar artery territory. F: On day 10, MRI demonstrated another new ischemic infarction, this time in the right pons and involving the medial longitudinal fasciculus. ADC: apparent diffusion coefficient, DWI: diffusion-weighted imaging, MRA: magnetic resonance angiography, MRI: magnetic resonance imaging, PCA: posterior cerebral artery, PICA: posterior inferior cerebellar artery.

artery (PICA) territory but normal-appearing vessels on $\mathrm{MR}$ angiography (MRA) (Fig. 1B). A diagnosis of acute ischemic stroke in the left PICA territory was made, and clopidogrel plus aspirin were administered to the patient.

On day 3 the patient was referred to our hospital for further etiological workups. The findings of a neurologic examination performed on arrival were normal except for a mild gait imbalance. The patient had no constitutional symptoms including fever, night sweats, fatigue, anorexia, or weight loss. The results of vascular studies, including MRA and computed tomography (CT) angiography, were unremarkable. Electrocardiography, 24-hr telemonitoring, transcranial Doppler shunt test, and coronary CT angiography revealed no abnormalities. The results of laboratory tests, including routine blood tests, inflammatory markers (erythrocyte sedimentation rate and C-reactive protein), thyroid function test, and autoantibodies, were normal. Hemostatic markers of prothrombotic tendency, including antiphospholipid antibodies (anticardiolipid antibody, lupus anticoagulant, dilute Russell's viper venom time, and $\beta 2$-glycoprotein-1 antibody), functional activity of antithrombin III, and proteins $\mathrm{C}$ and $\mathrm{S}$, were unremarkable, as were serological tests for autoimmune diseases, except for a mildly increased rheumatoid factor $(53.2 \mathrm{IU} / \mathrm{mL}$, reference range $0-14 \mathrm{IU} / \mathrm{mL}$ ). A provisional etiological diagnosis of 'stroke of undetermined etiology'3 was made, and the patient was treated using dual antiplatelet (aspirin and clopidogrel) and atorvastatin (20 mg) therapy.

On day 6 the patient complained about a visual-field abnormality. A neurologic examination revealed right-lower quadrantanopia. Diffusion-weighted imaging (DWI) and the apparent diffusion coefficient revealed a newly developed focal ischemic lesion in the left PCA territory (Fig. 1C). The results of follow-up MRA (Fig. 1D) and perfusion-weighted imaging were normal. In addition to antiplatelet treatment, dose-adjusted warfarin was started to prevent further embolic stroke. On day 7 the patient experienced a sudden exacerbation of vertigo and walking difficulty. A neurologic examination revealed ataxia of the right arm and leg, and DWI revealed a new ischemic lesion in the right superior cerebellar artery territory (Fig. 1E). Transfemoral cerebral angiography (TFCA) 
was performed, which revealed no arterial abnormalities, including in the vertebrobasilar system. On day 10 the patient presented with dysarthria and binocular diplopia, and exhibited medial gaze limitation of his right eye. Another DWI scan demonstrated a new ischemic lesion in the right pons involving the medial longitudinal fasciculus (Fig. 1F), and his prothrombin-time international normalized ratio was 2.29.

Intracranial arterial wall imaging of the vertebral artery and basilar artery (BA) was performed using a high-resolution, 3-tesla, contrast-enhanced MRI apparatus (Achieva, Philips Medical System, Best, The Netherlands). The arterial wall imaging protocol consisted of precontrast axial T1-, T2-, and proton-density-weighted (PD) images, and postcontrast axial and three-dimensional T1-weighted images. T1-weighted MRI was performed using a three-dimensional spin echo sequence with the following parameters: repetition time (TR)/echo time $(\mathrm{TE})=450 \mathrm{~ms} / 18 \mathrm{~ms}$, field of view $=170 \mathrm{~mm}$, matrix size $=$ $704 \times 704$, voxel size $=0.241 \times 0.241 \mathrm{~mm}^{2}$, echo train length $=10$, number of signal averages $=1$, and slice thickness $=0.5 \mathrm{~mm}$. The parameters used for the PD images were $\mathrm{TR} / \mathrm{TE}=2000$ $\mathrm{ms} / 32 \mathrm{~ms}$, field of view $=100 \mathrm{~mm}$, matrix size $=200 \times 200$, number of signal averages $=2$, and slice thickness $=1 \mathrm{~mm}$. The black-blood technique with preregional 80 -mm-thick saturation pulses to saturate the incoming arterial flow was used to visualize the vessel walls. Imaging analysis was performed by a neuroradiologist (J.W.C.) who was blinded to the clinical information. Axial PD and enhanced T1-weighted images demonstrated eccentric vessel-wall thickness with enhancement of the proximal BA (Fig. 2A).

An examination of the cerebrospinal fluid (CSF) revealed mild inflammation: white blood cell $=5 / \mu \mathrm{L}$, lymphocytes $=$ $74 \%$, red blood cell $=10 / \mu \mathrm{L}$, protein $=80.3 \mathrm{mg} / \mathrm{dL}$, and $\mathrm{CSF}$ glucose $=88 \mathrm{mg} / \mathrm{dL}$ ( $\mathrm{s}$-glucose $=147 \mathrm{mg} / \mathrm{dL})$. The patient was diagnosed as having recurrent ischemic stroke due to PACNS. High-dose intravenous steroid therapy ( $1 \mathrm{~g}$ /day for 3 days) was started followed by oral prednisolone. After starting the high-dose steroid therapy there was no stroke recurrence, and the patient gradually improved.

Repeated intracranial arterial wall imaging with high-resolution, 3-tesla, contrast-enhanced MRI performed 1 month after steroid therapy revealed decreased wall thickness and the nearly complete disappearance of contrast enhancement in the proximal BA (Fig. 2B). The patient's overall condition had improved relative to his last recurrent ischemic stroke. His residual deficits consisted of mild medial gaze limitation of the right eye, dysarthria, and ataxia of the right limbs. The patient was discharged and there was no recurrence while tapering oral steroid therapy.

\section{Discussion}

We have described herein a case of recurrent cerebral infarctions due to PACNS diagnosed by intracranial arterial wall imaging with high-resolution, 3-tesla, contrast-enhanced MRI. Although the TFCA and MRA findings of our patient were normal, several of the following features did support the diagnosis of PACNS in our patient. First, MRI may directly demonstrate inflammation of vessel walls, probably with a high diagnostic accuracy. ${ }^{4}$ Our patient presented with recurrent ischemic stroke only in the posterior circulation territory, and the arterial wall MRI revealed diffuse wall thickening and contrast enhancement of the BA. Second, dramatic clinical and radiological responses to steroid therapy favor a diagnosis of CNS angiitis. Premature atherosclerosis was evi-
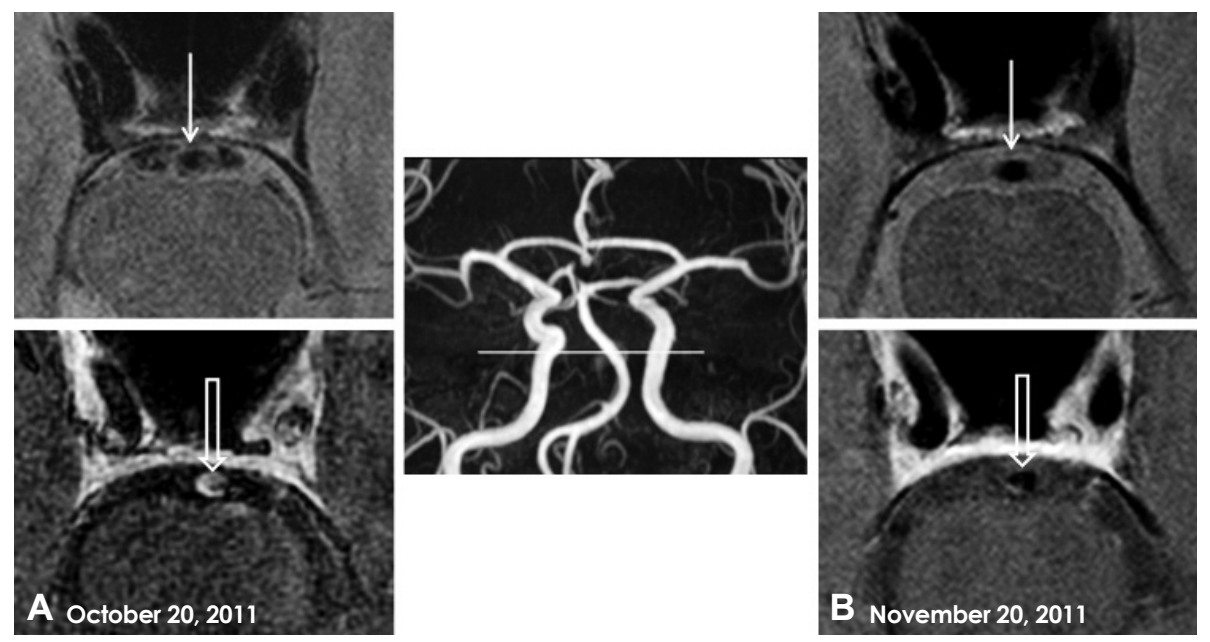

Fig. 2. Intracranial arterial wall imaging using high-resolution, 3-tesla, contrast-enhanced MRI. A: Axial PD image (upper) showing eccentric vessel-wall thickness (closed arrow), and a three-dimensional postcontrast T1-weighted image (lower) demonstrating concentric enhancement of the proximal BA wall (open arrow). B: On day 31, a repeat axial PD image (upper) and postcontrast T1-weighted image (lower) obtained after steroid treatment showed decreases in both wall thickness (closed arrow) and wall enhancement of the proximal BA (open arrow). BA: basilar artery, MRI: magnetic resonance imaging, PD: proton-density-weighted. 
dent as arterial wall thickening and contrast enhancement. However, the only vascular risk factor that the patient possessed was hyperlipidemia, and the CSF results revealed mild inflammation. This patient had very frequent recurrent strokes while receiving aggressive antithrombotic therapy, which stopped immediately after immunosuppressive treatment had started. Moreover, the arterial wall thickening and contrast enhancement disappeared after the steroid treatment.

Finally, the diagnostic tests excluded other conditions that mimic PACNS. Among such mimicking conditions, excluding infectious arteritis is important, especially varicella-zoster virus, human immunodeficiency virus, hepatitis-C viruses, and tuberculosis. In the present case, a polymerase chain reaction examination of the CSF for varicella zoster virus and Mycobacterium tuberculosis produced negative results. Serological tests yielded negative results for antihepatitis- $\mathrm{C}$ virus and human immunodeficiency virus antibody. A CNS tumor, especially intravascular lymphoproliferative disorder, could cause ischemic cerebrovascular events; however, CSF was negative for malignant cells. PACNS should be distinguished from secondary vasculitis of the CNS related to rheumatological disorders such as Wegener's granulomatosis, sarcoidosis, and Behçet's disease. The patient did not have any constitutional symptoms, skin lesions, or orogenital ulcers, and the results were negative for serological tests of rheumatological disorders including antinuclear antibody, antineutrophil cytoplasmic antibody, antiribonucleoprotein, anti-
Smith, anti-Sjögren's syndrome A, anti-Sjögren's syndrome B, and anti-Scl-70. Reversible cerebral vasoconstriction syndrome could mimic PACNS, but common features of this such as thunderclap headache, multifocal vasoconstriction, and parenchymal or cortical hemorrhagic lesions were not observed in our patient.

There are many modalities for the diagnosis of CNS angiitis, each of which has advantages and disadvantages (Fig. 3). Therefore, an appropriate diagnostic assessment should be made for patients for whom CNS angiitis is suspected. First, systemic symptoms may provide clues for the diagnosis of CNS angiitis. Secondary CNS angiitis related to systemic vasculitis usually presents with constitutional symptoms, ${ }^{5}$ and can therefore be diagnosed by careful history-taking and physical examination (e.g., oral and genital ulcers for Behçet's disease, and rash and photosensitivity for systemic lupus erythematosus). However, CNS angiitis presents with a variable clinical spectrum, and the constitutional symptoms lack sensitivity and specificity. Second, serological tests for autoimmune and inflammatory diseases such as systemic lupus erythematosus, rheumatoid arthritis, and Wegener's granulomatosis are valuable in the diagnosis of secondary CNS angiitis. However, false-positive and false-negative results are common in these tests, which makes them of little value in confirming or refuting PACNS. ${ }^{6}$ Acute-phase reactants such as the erythrocyte sedimentation rate and C-reactive protein are usually nondiagnostic. Third, an empirical immunosuppressive treatment can

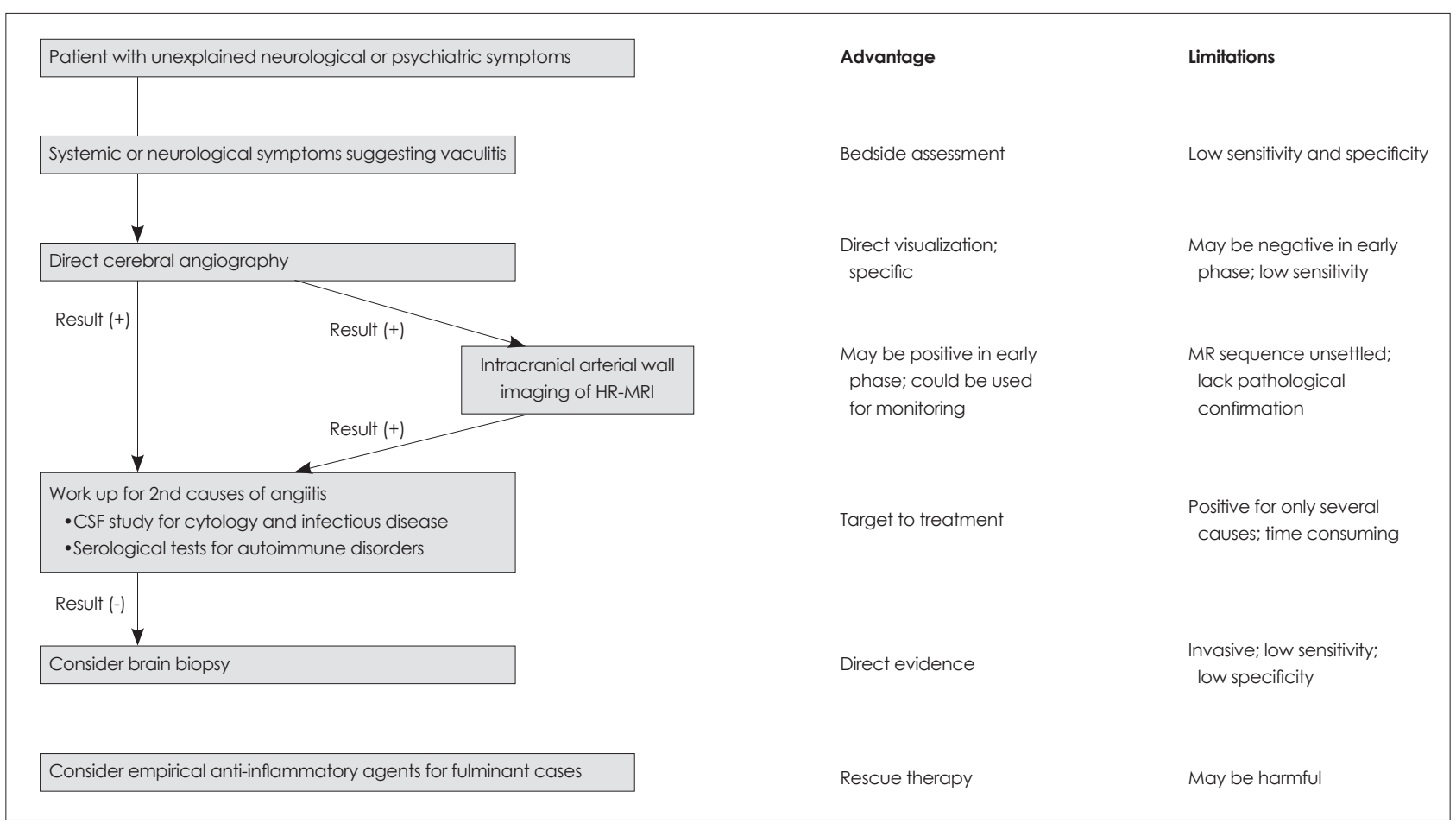

Fig. 3. Diagnostic tests for CNS angiitis and their advantages and disadvantages. CNS: central nervous system, CSF: cerebrospinal fluid, HR-MRI: high-resolution MRI. 
be considered in patients with fulminant symptoms. PACNS and secondary CNS angiitis related to systemic vasculitis may respond dramatically to immunosuppressive treatment. Highdose steroid therapy could have been a rescue therapy in the present case. The response would be different in other conditions, such as reversible cerebral vasoconstriction syndrome, neoplasm, and infection, and could even be harmful.

According to the current diagnostic criteria for PACNS, ${ }^{7}$ patients should have either classic angiographic abnormalities or histopathological features of angiitis within the CNS. A brain biopsy may provide direct evidence of CNS angiitis, and is valuable for the diagnosis of vasculitis when brain imaging is inconclusive and for the identification of the underlying etiology. However, the sensitivity of a brain biopsy for PACNS is low, at $<50 \%$, and false-negative biopsy findings reportedly occur in up to $25 \%$ of autopsy-documented cases. ${ }^{8}$ Therefore, noninvasive imaging modalities are important because a brain biopsy is further limited by the risk of complications and a low specificity. ${ }^{8}$

The established gold-standard imaging test for the diagnosis of vasculitis is angiography. Although MRA is the mainstay of documenting intracranial vascular stenoses, TFCA is required to investigate stenoses of medium-size and small arteries. MRA and TFCA neuroimaging results showing luminal changes are suggestive of CNS angiitis, especially if there is a vascular stenosis that is unlikely to be atherosclerotic, abnormal straightening and kinking of arteries caused by vesselwall induration, and multiple microaneurysms. ${ }^{4}$ However, even when a noninvasive or an invasive angiogram shows characteristic findings of CNS angiitis, they are far from specific since they are commonly encountered in nonvasculitis disorders such as atherosclerosis, radiation vasculopathy, infection, and vasospasm. ${ }^{9}$ More importantly, luminal changes may not be evident in the early phase of CNS vasculitis. The usefulness of high-resolution MRI over MRA for improved visualization of vessel-wall inflammation with abnormalities has been investigated. ${ }^{10}$ In the present case, repeated MRA and TFCA did not reveal any abnormalities, which suggests the limitation of current diagnostic criteria in the diagnosis of PACNS.

Cross-sectional imaging can be used to image the vesselwall changes associated with vasculitis. Intracranial arterial wall imaging with high-resolution, 3-tesla, contrast-enhanced MRI can complement the above-mentioned shortcomings, by visualizing vessel-wall inflammation directly (Fig. 2). In patients with Takayasu's arteritis, changes in the aorta such as wall thickening (>3-4 mm) and enhancement on contrastenhanced MRA and CT angiography have been used to assess the disease activity of aortitis. ${ }^{11}$ Although less is known about high-resolution MRI of vessel walls in patients with CNS vasculitis, ${ }^{4,12}$ vessel-wall thickening with contrast enhancement would be a direct imaging sign of CNS angiitis. ${ }^{4,13}$
As seen in the present case, high-resolution MRI can also be used to monitor the effects of treatment. The reversal of enhancement in arterial wall imaging could suggest a state of remission. However, since the volume coverage of high-resolution imaging is usually small due to scan-time limitations and patient movement, clinicians must identify and then target the field of vessel-wall MRI before performing the scan.

Since the first diagnostic criteria for PACNS were promoted by Calabrese and Mallek ${ }^{7}$ in the 1980s, valuable diagnostic modalities have been developed, and there has been a preponderance of case reports providing increasingly detailed and enriched clinical and pathological information. However, the prevalence of PACNS may still be underestimated. The present case emphasizes the importance of wall imaging in the diagnosis of PACNS.

\section{Conflicts of Interest}

The authors have no financial conflicts of interest.

\section{Acknowledgements}

This study was supported by the Korean Healthcare Technology R\&D Project, Ministry of Health \& Welfare (Grant no. A110208).

\section{REFERENCES}

1. Salvarani C, Brown RD Jr, Calamia KT, Christianson TJ, Weigand SD, Miller DV, et al. Primary central nervous system vasculitis: analysis of 101 patients. Ann Neurol 2007;62:442-451.

2. Hajj-Ali RA, Singhal AB, Benseler S, Molloy E, Calabrese LH. Primary angiitis of the CNS. Lancet Neurol 2011;10:561-572.

3. Adams HP Jr, Bendixen BH, Kappelle LJ, Biller J, Love BB, Gordon DL, et al. Classification of subtype of acute ischemic stroke. Definitions for use in a multicenter clinical trial. TOAST. Trial of Org 10172 in Acute Stroke Treatment. Stroke 1993;24:35-41.

4. Küker W. Cerebral vasculitis: imaging signs revisited. Neuroradiology 2007;49:471-479.

5. Molloy ES, Langford CA. Vasculitis mimics. Curr Opin Rheumatol 2008;20:29-34.

6. Scolding NJ. Central nervous system vasculitis. Semin Immunopathol 2009;31:527-536.

7. Calabrese LH, Mallek JA. Primary angiitis of the central nervous system. Report of 8 new cases, review of the literature, and proposal for diagnostic criteria. Medicine (Baltimore) 1988;67:20-39.

8. Cupps TR, Moore PM, Fauci AS. Isolated angiitis of the central nervous system. Prospective diagnostic and therapeutic experience. Am J Med 1983;74:97-105.

9. Alhalabi M, Moore PM. Serial angiography in isolated angiitis of the central nervous system. Neurology 1994;44:1221-1226.

10. Küker W, Gaertner S, Nagele T, Dopfer C, Schoning M, Fiehler J, et al. Vessel wall contrast enhancement: a diagnostic sign of cerebral vasculitis. Cerebrovasc Dis 2008;26:23-29.

11. Kissin EY, Merkel PA. Diagnostic imaging in Takayasu arteritis. Curr Opin Rheumatol 2004;16:31-37.

12. Swartz RH, Bhuta SS, Farb RI, Agid R, Willinsky RA, Terbrugge KG, et al. Intracranial arterial wall imaging using high-resolution 3-tesla contrast-enhanced MRI. Neurology 2009;72:627-634.

13. Saam T, Habs M, Pollatos O, Cyran C, Pfefferkorn T, Dichgans M, et al. High-resolution black-blood contrast-enhanced T1 weighted images for the diagnosis and follow-up of intracranial arteritis. $\mathrm{Br} J$ Radiol 2010;83:e182-e184. 